\title{
Phyllosphere Microflora and Soil Dehydrogenase Activity as Influenced by Weed Management Practices
}

\author{
Tasmiya Kowser $^{1 *}$, A.S. Halepyati ${ }^{1}$, B.M. Chittapur ${ }^{2}$, A.S. Channabasavanna ${ }^{3}$, \\ I. Shanker Goud ${ }^{4}$ and Basave Gowda ${ }^{5}$
}

\author{
${ }^{1}$ Department of Agronomy, College of Agriculture, Raichur 584104, Karnataka, India \\ ${ }^{2}$ Agricultural Extension Education Centre, Koppal-583227, UAS Raichur, Karnataka, India \\ ${ }^{3}$ Department of Agronomy, UAS Raichur 584104, Karnataka, India \\ ${ }^{4}$ Directorate of Research, UAS Raichur 584104, Karnataka, India \\ ${ }^{5}$ Seeds, UAS Raichur 584104, Karnataka, India \\ *Corresponding author
}

A B S T R A C T

\begin{tabular}{|l|}
\hline Ke y w o r d s \\
Castor, \\
$\begin{array}{l}\text { Dehydrogenase } \\
\text { activity, Weed } \\
\text { management, Bacteria } \\
\text { and Phyllosphere } \\
\text { micro flora. }\end{array}$ \\
\hline Article Info \\
\hline $\begin{array}{l}\text { Accepted: } \\
\text { 19 October } 2017 \\
\text { Available Online: } \\
\text { 10 December } 2017\end{array}$ \\
\hline
\end{tabular}

Keywords

Castor,

Dehydrogenas

and Phyllosphere

micro flora.

10 December 2017

Field experiment was conducted on weed management practices in castor at the Agricultural College Farm, UAS, Raichur, during kharif 2015-16 and 2016-17 in the clay soil. Pooled mean of two years indicated that among the weed management treatments, application of pendimethalin 38.7\% CS @ $1000 \mathrm{~g}$ a.i. ha ${ }^{-1}$ (PRE) fb imazethapyr 10\% SL @ $75 \mathrm{~g}$ a.i. $\mathrm{ha}^{-1}$ (POE) recorded significantly higher herbicide efficiency index $(0.0451)$, dehydrogenase activity (23.37 $\mu \mathrm{g}$ TPF formed $\mathrm{g}^{-1}$ soil $\left.\mathrm{hr}^{-1}\right)$ at 60 DAS. Whereas bacterial (12.25 per plate) and fungal population (8.61 per plate) at 15 DAH application was significantly higher with pendimethalin $38.7 \% \mathrm{CS} @ 1000 \mathrm{~g}$ a.i. ha ${ }^{-1}(\mathrm{PRE})+2$ intercultivations at 30 and 60 DAS compared to other herbicidal treatments and was found at par with the application of pendimethalin 38.7\% CS @ $1000 \mathrm{~g}$ a.i. ha ${ }^{-1}$ (PRE) fb imazethapyr 10\% SL @ $75 \mathrm{~g}$ a.i. $\mathrm{ha}^{-1}$ (POE) at $30 \mathrm{DAS}+\mathrm{IC}$ at 60 DAS (12.04 and 8.40 per plate).

\section{Introduction}

Castor (Ricinus communis L.) is valuable nonedible oil seed crop playing an important role in agriculture economy. Castor oil has diversified uses and has great value of foreign trade. Leaves of castor are rich source of protein (23 to $25 \%$ ) and used for ericulture. Castor cake a byproduct is used as organic manuring. Castor stalks are useful in manufacturing paper, card-board and also widely used as a fuel. Its hull is used as manure after decomposition. Several measures have been suggested to control the weeds. Hand weeding and inter-culturing are effective, but always associated with regeneration of weeds and requires frequent operations, which makes this practice sometimes costly and also not feasible all times due to unavailability of labours and implements. The use of herbicide in crop land generally results in increase in crop yield and reduces production cost. Herbicides used alone or in combination with other weed control method reduced the crop-weed competition and the risk of weeds growing in 
adverse weather or soil condition that would hinder the use of more traditional weed control methods. Modern agriculture depends on herbicides for the control of weeds in crops to increase yield. Herbicides are therefore, very important to agriculture but their heavy use may lead to pollution of soil environment, ground waters and environment. Further, herbicides application also found to influence phyllosphere microflora and can cause changes in microbial community function and soil dehydrogenase activity.

\section{Materials and Methods}

A field experiment was conducted on herbicidal effect on herbicide efficiency index, phyllosphere micro flora and soil dehydrogenase activity at different crop growth stages in castor during kharif 2015-16 and 2016-17 on medium deep black clay soils at the Agricultural College Farm, UAS, Raichur, Karnataka. The experiment was laid out in Randomised Complete Block Design.

The recommended dose of fertilizers 75:50:25 $\mathrm{kg} \mathrm{N}: \mathrm{P}_{2} \mathrm{O}_{5}: \mathrm{K}_{2} \mathrm{O}$ ha $^{-1}$ were applied in the form of urea, diammonium phosphate (DAP) and muriate of potash (MOP) The experimental soil was alkaline in reaction $(\mathrm{pH} 8.3)$ and $(\mathrm{EC}$ $\left.0.32 \mathrm{dSm}^{-1}\right)$. It was also low in available nitrogen $\left(226 \mathrm{~kg} \mathrm{ha}^{-1}\right)$ by Subbiah and Asija, 1956 , medium in available phosphorus $(29 \mathrm{~kg}$ $\left.\mathrm{ha}^{-1}\right)$, potash $\left(320 \mathrm{~kg} \mathrm{ha}^{-1}\right)$ and sulphur (18 ppm) by Jackson, 1973. Enumeration of phyllosphere microflora was made by leaf impression method with isolation of microorganisms (Dubey and Maheshwari, 2008). Dehydrogenase activity in the soil sample was determined by colorimetric determination of TPF produced from the TTC in soils by Assay method as described by Casida et al., (1964) at a wave length of 485 $\mathrm{nm}$. The recommended packages of practices were followed. Herbicide efficiency index indicates the weed killing potential of different herbicide treatments and their phytotoxicity on the crop (Devasenapathy et al., 2008).

$(\mathrm{Yt}-\mathrm{Yc}) / \mathrm{Yc}$

Herbicide efficiency index = -

$(\mathrm{DMt} / \mathrm{DMc}) \times 100$

Where,

$\mathrm{Yt}=$ Yield from treated plot

Yc $=$ Yield from control plot

DMt $=$ Dry matter of weed in treated plot

$\mathrm{DMc}=$ Dry matter of weed in control

\section{Results and Discussion}

\section{Herbicide efficiency index}

Herbicide efficiency index indicate the weed killing potential of different herbicide was significant at all the growth stages viz., 20, 40, 60 DAS and at harvest. The herbicide efficiency index at 40 DAS was significantly higher in pendimethalin (38.7\% CS) @ 1000 $\mathrm{g}$ a.i. ha ${ }^{-1} \mathrm{fb}$ imazethapyr (10\% SL) @ $75 \mathrm{~g}$ a.i. $\mathrm{ha}^{-1}$ at $30 \mathrm{DAS}+\mathrm{IC}$ at 60 DAS (0.0477), pendimethalin (38.7\% CS) @ 1000 g a.i. $\mathrm{ha}^{-1}+\mathrm{IC}$ at 30 and 60 DAS (0.0298), pendimethalin $(38.7 \% \mathrm{CS}) @ 1000 \mathrm{~g}$ a.i. ha ${ }^{-1}$ fb quizalofop-ethyl (5\% EC) @ $50 \mathrm{~g}$ a.i. ha ${ }^{1}$ at 30 DAS + IC at 60 DAS (0.0213) and clomazone (20\% EC) @ 750 g a.i. ha ${ }^{-1} \mathrm{fb}$ imazethapyr (10\% SL) @ $75 \mathrm{~g}$ a.i. ha ${ }^{-1}$ at 30 DAS + IC at 60 DAS (0.0173) at 40 DAS. Significantly lower HEI was recorded in post emergence application of chlorimuron ethyl (25\% WP) @ $15 \mathrm{~g}$ a.i. $\mathrm{ha}^{-1}$ at 15-20 DAS + IC at 60 DAS (0.0033), quizalofop-ethyl (5\% EC) @ $50 \mathrm{~g}$ a.i. ha ${ }^{-1}$ at $30 \mathrm{DAS}+\mathrm{IC}$ at 60 DAS (0.0047) and imazethapyr (10\% SL) @ $75 \mathrm{~g}$ a.i. ha ${ }^{-1}$ at $30 \mathrm{DAS}+\mathrm{IC}$ at $60 \mathrm{DAS}$ (0.0062) (Table 1). This might be due to sequential application of herbicides which managed weeds from early period of crop and found to be more efficient in killing of weeds than the post emergent application of herbicides. 
Table.1 Herbicide efficiency index in castor at different growth stages as influenced by weed management practices

\begin{tabular}{|c|c|c|c|c|c|c|c|c|c|c|c|c|}
\hline \multirow{3}{*}{ Treatment } & \multicolumn{12}{|c|}{ Herbicide efficiency index } \\
\hline & \multicolumn{3}{|c|}{20 DAS } & \multicolumn{3}{|c|}{40 DAS } & \multicolumn{3}{|c|}{$60 \mathrm{DAS}$} & \multicolumn{3}{|c|}{ At harvest } \\
\hline & 2015 & 2016 & Pooled & 2015 & 2016 & Pooled & 2015 & 2016 & Pooled & 2015 & 2016 & Pooled \\
\hline $\mathrm{T}_{1}$ & 0.0322 & 0.0312 & 0.0316 & 0.0305 & 0.0292 & 0.0298 & 0.0392 & 0.0346 & 0.0367 & 0.0268 & 0.0244 & 0.0256 \\
\hline $\mathrm{T}_{2}$ & 0.0056 & 0.0058 & 0.0057 & 0.0095 & 0.0091 & 0.0093 & 0.0115 & 0.0111 & 0.0112 & 0.0104 & 0.0095 & 0.0099 \\
\hline $\mathrm{T}_{3}$ & 0.0113 & 0.0116 & 0.0115 & 0.0139 & 0.0136 & 0.0138 & 0.0196 & 0.0185 & 0.0191 & 0.0163 & 0.0150 & 0.0156 \\
\hline $\mathrm{T}_{4}$ & 0.0027 & 0.0021 & 0.0024 & 0.0053 & 0.0041 & 0.0047 & 0.0052 & 0.0041 & 0.0047 & 0.0053 & 0.0039 & 0.0046 \\
\hline $\mathrm{T}_{5}$ & 0.0022 & 0.0014 & & & 0.0025 & 0.0033 & 0.0037 & & & 0.0043 & & 0.0034 \\
\hline $\mathrm{T}_{6}$ & 0.0033 & 0.0026 & 0.0029 & 0.0067 & 0.0056 & 0.0062 & 0.0067 & 0.0054 & 0.0061 & 0.0064 & 0.0047 & 0.0056 \\
\hline $\mathrm{T}_{7}$ & 0.0281 & 0.0244 & 0.0263 & 0.0239 & 0.0191 & & 0.0296 & 0.0266 & 0.0281 & 0.0241 & 0.0189 & 0.0215 \\
\hline $\mathrm{T}_{8}$ & 0.0131 & 0.0096 & 0.0115 & 0.0078 & 0.0059 & 0.0069 & 0.0092 & 0.0067 & 0.0079 & 0.0082 & 0.0060 & 0.0071 \\
\hline $\mathrm{T}_{9}$ & 0.0436 & 0.0402 & 0.0415 & 0.0490 & 0.0465 & 0.0477 & 0.0471 & 0.0433 & 0.0451 & 0.0306 & 0.0274 & 0.0290 \\
\hline $\mathrm{T}_{10}$ & 0.0091 & 0.0096 & 0.0094 & 0.0110 & 0.0110 & 0.0110 & 0.0145 & 0.0131 & 0.0138 & 0.0131 & 0.0120 & 0.0125 \\
\hline $\mathrm{T}_{11}$ & 0.0062 & 0.0051 & 0.0057 & 0.0074 & 0.0059 & 0.0067 & 0.0085 & 0.0067 & 0.0076 & 0.0075 & 0.0056 & 0.0066 \\
\hline $\mathrm{T}_{12}$ & 0.0132 & 0.0123 & & 0.0183 & 0.0164 & 0.0173 & 0.0233 & 0.0205 & & 0.0191 & 0.0161 & 0.0175 \\
\hline $\mathrm{T}_{13}$ & 0.0000 & 0.0000 & 0.0000 & 0.0000 & 0.0000 & 0.0000 & 0.0000 & 0.0000 & 0.0000 & 0.0000 & 0.0000 & 0.0000 \\
\hline $\mathrm{T}_{14}$ & 0.0000 & 0.0000 & 0.0000 & 0.0000 & 0.0000 & 0.0000 & 0.0000 & 0.0000 & 0.0000 & 0.0000 & 0.0000 & 0.0000 \\
\hline S.Em. \pm & 0.003 & 0.003 & 0.007 & 0.003 & 0.003 & 0.01 & 0.003 & 0.003 & 0.01 & 0.003 & 0.003 & 0.003 \\
\hline C.D. at $5 \%$ & 0.009 & 0.009 & 0.019 & 0.009 & 0.009 & 0.02 & 0.009 & 0.009 & 0.03 & 0.009 & 0.009 & 0.009 \\
\hline
\end{tabular}

$\mathrm{T}_{1}$ : Pendimethalin 38.7\% CS @ $1000 \mathrm{~g}$ a.i. ha ${ }^{-1}(\mathrm{PRE})+\mathrm{IC}$ at 30 and 60 DAS

$\mathrm{T}_{2}$ : Alachlor 50\% EC @ $1000 \mathrm{~g}$ a.i. ha ${ }^{-1}(\mathrm{PRE})+\mathrm{IC}$ at 30 and 60 DAS

$\mathrm{T}_{3}$ : Clomazone 20\% EC @ $750 \mathrm{~g}$ a.i. ha ${ }^{-1}(\mathrm{PRE})+\mathrm{IC}$ at 30 and 60 DAS

$\mathrm{T}_{4}$ Quizalofop-ethyl 5\% EC @ $50 \mathrm{~g}$ a.i. ha ${ }^{1}$ (POE) at 30 DAS $+\mathrm{IC}$ at $60 \mathrm{DAS}$

$\mathrm{T}_{5}$ : Chlorimuron ethyl 25\% WP @ $15 \mathrm{~g}$ a.i. $\mathrm{ha}^{-1}$ (EPOE) at 1520 DAS + IC at 60 DAS

$\mathrm{T}_{6}$ : Imazethapyr 10\% SL @ $75 \mathrm{~g}$ a.i. ha ${ }^{-1}$ (POE)at $30 \mathrm{DAS}+$ IC at 60 DAS

$\mathrm{T}_{7}$ : Pendimethalin $38.7 \%$ CS @ 1000 g a.i. ha ${ }^{-1}$ (PRE) fb Quizalofop-ethyl (5\% EC) (POE) @ 50 g a.i. ha ${ }^{1}$ at 30 DAS + IC at 60 DAS
$\mathrm{T}_{8}$ : Pendimethalin 38.7\% CS @ $1000 \mathrm{~g}$ a.i. ha $^{-1}$ (PRE) fb Chlorimuron ethyl 25\% WP @ $15 \mathrm{~g}$ a.i. ha (EPOE) at 15-20 DAS + IC at $60 \mathrm{DAS}$

$\mathrm{T}_{9}$ : Pendimethalin 38.7\% CS @ 1000 g a.i. ha ${ }^{-1}$ (PRE) fb Imazethapyr 10\% SL @ 75 g a.i. ha ${ }^{-1}$ (POE) at 30 DAS + IC at 60 DAS

$\mathrm{T}_{10}$ : Clomazone 20\% EC @ $750 \mathrm{~g}$ a.i. ha ${ }^{-1}$ (PRE) fb Quizalofop-ethyl 5\% EC @ $50 \mathrm{~g}$ a.i. ha ${ }^{1}$ (POE) at $30 \mathrm{DAS}+\mathrm{IC}$ at $60 \mathrm{DAS}$

$\mathrm{T}_{11}$ : Clomazone 20\% EC @ $750 \mathrm{~g}$ a.i. ha ${ }^{-1}$ (PRE) fb Chlorimuron ethyl 25\% WP @ $15 \mathrm{~g}$ a.i. ha ${ }^{-1}$ (EPOE) at 15-20 DAS + IC at 60 DAS

$\mathrm{T}_{12}$ : Clomazone $20 \%$ EC @ $750 \mathrm{~g}$ a.i. ha ${ }^{-1}$ (PRE) fb Imazethapyr $(10 \% \mathrm{SL})$ (POE) @ $75 \mathrm{~g}$ a.i. ha ${ }^{-1}$ at 30 DAS + IC at 60 DAS

$\mathrm{T}_{13}$ : Weedy check

$\mathrm{T}_{14}:$ Weed free check 
Table.2 Bacterial and fungal population at 45 DAS in phyllosphere of castor as influenced by weed management practices

\begin{tabular}{|c|c|c|c|c|c|c|}
\hline \multirow{2}{*}{ Treatment } & \multicolumn{3}{|c|}{ Bacterial population per plate } & \multicolumn{3}{|c|}{ Fungal population per plate } \\
\hline & 2015 & 2016 & Pooled & 2015 & 2016 & Pooled \\
\hline $\mathrm{T}_{1}$ & 12.00 & 12.50 & 12.25 & 8.37 & 8.85 & 8.61 \\
\hline $\mathrm{T}_{2}$ & 10.40 & 11.02 & 10.71 & 6.52 & 7.00 & 6.76 \\
\hline $\mathrm{T}_{3}$ & 10.86 & 11.38 & 11.12 & 7.27 & 7.87 & 7.57 \\
\hline $\mathrm{T}_{4}$ & 9.13 & 10.00 & 9.57 & 5.55 & 6.00 & 5.78 \\
\hline $\mathrm{T}_{5}$ & 8.36 & 9.61 & 8.99 & 5.00 & 5.73 & 5.37 \\
\hline $\mathrm{T}_{6}$ & 9.45 & 10.14 & 9.80 & 5.78 & 6.33 & 6.06 \\
\hline $\mathrm{T}_{7}$ & 11.39 & 12.00 & 11.70 & 8.00 & 8.33 & 8.17 \\
\hline $\mathrm{T}_{8}$ & 10.07 & 10.90 & 10.48 & 6.25 & 6.83 & 6.54 \\
\hline $\mathrm{T}_{9}$ & 11.76 & 12.32 & 12.04 & 8.33 & 8.47 & 8.40 \\
\hline $\mathrm{T}_{10}$ & 10.60 & 11.14 & 10.87 & 7.00 & 7.65 & 7.33 \\
\hline $\mathrm{T}_{11}$ & 9.95 & 10.49 & 10.22 & 6.00 & 6.50 & 6.25 \\
\hline $\mathrm{T}_{12}$ & 11.25 & 11.96 & 11.61 & 7.82 & 8.00 & 7.91 \\
\hline $\mathrm{T}_{13}$ & 12.16 & 12.69 & 12.43 & 8.48 & 9.00 & 8.74 \\
\hline $\mathrm{T}_{14}$ & 12.34 & 12.88 & 12.61 & 8.62 & 9.25 & 8.93 \\
\hline S.Em. \pm & 0.12 & 0.12 & 0.08 & 0.12 & 0.17 & 0.09 \\
\hline C.D. at 5\% & 0.36 & 0.36 & 0.25 & 0.36 & 0.51 & 0.27 \\
\hline
\end{tabular}

$\mathrm{T}_{1}$ : Pendimethalin $38.7 \% \mathrm{CS} @ 1000 \mathrm{~g}$ a.i. ha ${ }^{-1}(\mathrm{PRE})+\mathrm{IC}$ at 30 and 60 DAS

$\mathrm{T}_{2}$ : Alachlor 50\% EC @ $1000 \mathrm{~g}$ a.i. ha ${ }^{-1}(\mathrm{PRE})+\mathrm{IC}$ at 30 and 60 DAS

$\mathrm{T}_{8}$ : Pendimethalin 38.7\% CS @ $1000 \mathrm{~g}$ a.i. ha ${ }^{-1}$ (PRE) fb Chlorimuron ethyl 25\% WP @ $15 \mathrm{~g}$ a.i. ha ${ }^{-1}$ (EPOE) at 15-20 DAS + IC at 60 DAS

$\mathrm{T}_{9}$ : Pendimethalin 38.7\% CS @ 1000 g a.i. ha ${ }^{-1}$ (PRE) fb Imazethapyr 10\% SL @ 75 g a.i. ha ${ }^{-1}$ (POE) at $30 \mathrm{DAS}+\mathrm{IC}$ at $60 \mathrm{DAS}$

$\mathrm{T}_{3}$ : Clomazone $20 \%$ EC @ $750 \mathrm{~g}$ a.i. ha ${ }^{-1}(\mathrm{PRE})+\mathrm{IC}$ at 30 and 60 DAS

$\mathrm{T}_{10}$ : Clomazone 20\% EC @ $750 \mathrm{~g}$ a.i. ha ${ }^{-1}$ (PRE) fb Quizalofop-ethyl 5\% EC @ $50 \mathrm{~g}$ a.i. ha ${ }^{1}$ (POE) at $30 \mathrm{DAS}+\mathrm{IC}$ at $60 \mathrm{DAS}$

$\mathrm{T}_{4}$ Quizalofop-ethyl 5\% EC @ $50 \mathrm{~g}$ a.i. ha ${ }^{1}$ (POE) at $30 \mathrm{DAS}+$ IC at 60 DAS

$\mathrm{T}_{11}$ : Clomazone 20\% EC @ $750 \mathrm{~g}$ a.i. ha ${ }^{-1}$ (PRE) fb Chlorimuron ethyl 25\% WP @ $15 \mathrm{~g}$ a.i. ha ${ }^{-1}$ (EPOE) at 15-20 DAS + IC at 60 DAS

$\mathrm{T}_{5}$ : Chlorimuron ethyl 25\% WP @ $15 \mathrm{~g}$ a.i. $\mathrm{ha}^{-1}$ (EPOE) at 15

$\mathrm{T}_{12}$ : Clomazone 20\% EC @ $750 \mathrm{~g}$ a.i. ha ${ }^{-1}$ (PRE) fb Imazethapyr (10\% SL) (POE) @ $75 \mathrm{~g}$ a.i. ha ${ }^{-1}$ at 30 20 DAS + IC at 60 DAS

DAS + IC at 60 DAS

$\mathrm{T}_{6}$ : Imazethapyr 10\% SL @ 75 g a.i. ha ${ }^{-1}$ (POE)at 30 DAS + $\quad \mathrm{T}_{13}$ : Weedy check

IC at 60 DAS

$\mathrm{T}_{7}$ : Pendimethalin $38.7 \%$ CS @ $1000 \mathrm{~g}$ a.i. ha $^{-1}$ (PRE) fb

Quizalofop-ethyl (5\% EC) (POE) @ $50 \mathrm{~g}$ a.i. ha ${ }^{1}$ at $30 \mathrm{DAS}+$

$\mathrm{T}_{14}$ : Weed free check

IC at 60 DAS 
Table.3 Dehydrogenase activity in soil at different growth stages of castor as influenced by weed management practices

\begin{tabular}{|c|c|c|c|c|c|c|c|c|c|}
\hline \multirow{3}{*}{ Treatment } & \multicolumn{9}{|c|}{ Dehydrogenase activity $\left(\mu \mathrm{g}\right.$ TPF formed $\mathrm{g}^{-1}$ soil $\mathrm{hr}^{-1}$ ) } \\
\hline & \multicolumn{3}{|c|}{ Initial $\mu \mathrm{g}$ TPF formed $\mathrm{g}^{-1}$ soil $\mathrm{hr}^{-1}$} & \multicolumn{3}{|c|}{$\begin{array}{l}\text { At } 60 \text { DAS } \mu \mathrm{g} \text { TPF formed } \mathrm{g}^{-1} \text { soil } \\
\mathrm{hr}^{-1}\end{array}$} & \multicolumn{3}{|c|}{ At harvest $\mu \mathrm{g}$ TPF formed $\mathrm{g}^{-1}$ soi } \\
\hline & 2015 & 2016 & Pooled & 2015 & 2016 & Pooled & 2015 & 2016 & Pooled \\
\hline $\mathrm{T}_{1}$ & 20.20 & 18.56 & 19.38 & 24.34 & 22.08 & 23.21 & 18.56 & 16.45 & 17.51 \\
\hline $\mathrm{T}_{2}$ & 19.75 & 18.25 & 19.00 & 23.43 & 21.66 & 22.55 & 18.25 & 16.15 & 17.20 \\
\hline $\mathrm{T}_{3}$ & 19.88 & 18.44 & 19.16 & 23.50 & 21.83 & 22.67 & 18.44 & 16.35 & 17.40 \\
\hline $\mathrm{T}_{4}$ & 19.06 & 17.66 & 18.36 & 23.05 & 20.62 & 21.84 & 17.66 & 15.58 & 16.62 \\
\hline $\mathrm{T}_{5}$ & 19.00 & 17.64 & 18.32 & 23.03 & 20.19 & 21.61 & 17.64 & 15.50 & 16.57 \\
\hline $\mathrm{T}_{6}$ & 19.06 & 17.94 & 18.50 & 23.12 & 21.28 & 22.20 & 17.94 & 15.70 & 16.82 \\
\hline $\mathrm{T}_{7}$ & 20.00 & 18.51 & 19.26 & 24.28 & 22.04 & 23.16 & 18.51 & 16.43 & 17.47 \\
\hline $\mathrm{T}_{8}$ & 19.60 & 18.09 & 18.85 & 23.40 & 21.59 & 22.50 & 18.09 & 16.00 & 17.05 \\
\hline $\mathrm{T}_{9}$ & 20.28 & 18.55 & 19.42 & 24.52 & 22.21 & 23.37 & 18.55 & 16.55 & 17.55 \\
\hline $\mathrm{T}_{10}$ & 19.80 & 18.39 & 19.10 & 23.46 & 21.71 & 22.59 & 18.39 & 16.29 & 17.34 \\
\hline $\mathrm{T}_{11}$ & 19.28 & 18.00 & 18.64 & 23.19 & 21.34 & 22.27 & 18.00 & 15.86 & 16.93 \\
\hline $\mathrm{T}_{12}$ & 20.00 & 18.50 & 19.25 & 24.19 & 22.01 & 23.10 & 18.50 & 16.40 & 17.45 \\
\hline $\mathrm{T}_{13}$ & 20.40 & 18.77 & 19.59 & 24.22 & 22.22 & 23.22 & 18.77 & 16.57 & 17.67 \\
\hline $\mathrm{T}_{14}$ & 20.47 & 18.92 & 19.70 & 24.55 & 22.28 & 23.42 & 18.92 & 16.75 & 17.84 \\
\hline S.Em. \pm & 0.35 & 0.09 & 0.33 & 0.25 & 0.12 & 0.40 & 0.09 & 0.06 & 0.30 \\
\hline C.D. at 5\% & $\mathbf{N S}$ & 0.27 & 0.99 & 0.73 & $\mathbf{0 . 3 3}$ & 1.16 & 0.27 & 0.17 & 0.88 \\
\hline
\end{tabular}

$\mathrm{T}_{1}$ : Pendimethalin $38.7 \%$ CS @ $1000 \mathrm{~g}$ a.i. ha ${ }^{-1}$ (PRE) + IC at 30 and 60 DAS

$\mathrm{T}_{2}$ : Alachlor 50\% EC @ $1000 \mathrm{~g}$ a.i. ha ${ }^{-1}(\mathrm{PRE})+\mathrm{IC}$ at 30 and $60 \mathrm{DAS}$

$\mathrm{T}_{3}$ : Clomazone 20\% EC @ 750 g a.i. ha ${ }^{-1}(\mathrm{PRE})+\mathrm{IC}$ at 30 and 60 DAS

$\mathrm{T}_{4}$ Quizalofop-ethyl 5\% EC @ $50 \mathrm{~g}$ a.i. ha ${ }^{1}$ (POE) at $30 \mathrm{DAS}+\mathrm{IC}$ at 60 DAS

$\mathrm{T}_{5}$ : Chlorimuron ethyl 25\% WP @ 15 g a.i. ha ${ }^{-1}$ (EPOE) at 15-20 DAS + IC at 60 DAS

$\mathrm{T}_{6}$ : Imazethapyr 10\% SL @ 75 g a.i. ha $^{-1}$ (POE)at $30 \mathrm{DAS}+\mathrm{IC}$ at 60 DAS

$\mathrm{T}_{7}$ : Pendimethalin $38.7 \%$ CS @ $1000 \mathrm{~g}$ a.i. ha ${ }^{-1}$ (PRE) fb Quizalofopethyl (5\% EC) (POE) @ $50 \mathrm{~g}$ a.i. ha ${ }^{1}$ at $30 \mathrm{DAS}+\mathrm{IC}$ at $60 \mathrm{DAS}$
$\mathrm{T}_{8}$ : Pendimethalin 38.7\% CS @ $1000 \mathrm{~g}$ a.i. ha ${ }^{-1}$ (PRE) fb Chlorimuron ethyl 25\% WP @ $15 \mathrm{~g}$ a.i. ha ${ }^{-1}$ (EPOE) at 1520 DAS + IC at 60 DAS

T: Pendimethalin 38.7\% CS @ $1000 \mathrm{~g}$ a.i. $\mathrm{ha}^{-1}$ (PRE) fb Imazethapyr 10\% SL @ $75 \mathrm{~g}$ a.i. ha ${ }^{-1}$ (POE) at $30 \mathrm{DAS}+$ IC at 60 DAS

$\mathrm{T}_{10}$ : Clomazone 20\% EC @ 750 g a.i. ha ${ }^{-1}$ (PRE) fb Quizalofop-ethyl 5\% EC @ 50 g a.i. ha ${ }^{1}$ (POE) at 30 DAS + IC at 60 DAS

$\mathrm{T}_{11}$ : Clomazone 20\% EC @ $750 \mathrm{~g}$ a.i. ha ${ }^{-1}$ (PRE) fb Chlorimuron ethyl 25\% WP @ $15 \mathrm{~g}$ a.i. ha ${ }^{-1}$ (EPOE) at 15-20 DAS + IC at 60 DAS

$T_{12}$ : Clomazone 20\% EC @ 750 g a.i. ha ${ }^{-1}$ (PRE) fb Imazethapyr (10\% SL) (POE) @ 75 g a.i. ha ${ }^{-1}$ at 30 DAS + IC at 60 DAS

$\mathrm{T}_{13}$ : Weedy check

$\mathrm{T}_{14}$ : Weed free check 
Similarly these results were in conformity with finding by Malik et al., (2008), Gill et al., (2002) and Adhikari and Ghosh (2014).

\section{Phyllosphere microflora}

Modern agriculture depends on herbicides for the control of weeds in crops to increase yield. Herbicides are, therefore, very important to agriculture but their heavy use may lead to pollution of soil environment, ground waters and environment. Further, herbicides application also found to influence phyllosphere microflora and can cause changes in microbial community function. In the present study bacterial and fungal population varied significantly after herbicide application (DAH). There was little variation in the bacterial and fungal population among the weed control treatments. At $15 \mathrm{DAH}$, general rise in bacterial and fungal count per plate was observed in weed free check followed by weedy check as compared to the herbicide applied treatments (Table 2).

The data on bacterial population revealed that significantly higher population was recorded in absolute weed free check (12.61 per plate) followed by weedy check (12.43 per plate). Among the weedicide treatments, pendimethalin (38.7\% CS) @ $1000 \mathrm{~g}$ a.i. ha ${ }^{-1}$ + IC at 30 and 60 DAS (12.25 per plate) recorded higher bacterial population but it was found to be on par with pendimethalin (38.7\% CS) @ 1000 g a.i. ha ${ }^{-1}$ fb imazethapyr (10\% SL) @ $75 \mathrm{~g}$ a.i. ha ${ }^{-1}$ at $30 \mathrm{DAS}+\mathrm{IC}$ at 60 DAS (12.04 per plate). Significantly lower bacterial population was noticed in quizalofop-ethyl (5\% EC) @ $50 \mathrm{~g}$ a.i. $\mathrm{ha}^{-1}$ at 30 DAS + IC at 60 DAS (9.57 per plate) and chlorimuron ethyl (25\% WP) @ $15 \mathrm{~g}$ a.i. ha ${ }^{-1}$ at $15-20 \mathrm{DAS}+\mathrm{IC}$ at $60 \mathrm{DAS}$ (8.99 per plate).

Fungal population was significantly higher in weed free check (8.93 per plate) as compared to rest of the treatments. The next best treatment which recorded higher fungal population was weedy check ( 8.74 per plate). The treatments viz., pendimethalin (38.7\% CS) @ $1000 \mathrm{~g}$ a.i. ha ${ }^{-1}+\mathrm{IC}$ at 30 and 60 DAS (8.61 per plate) and pendimethalin (38.7\% CS) @ $1000 \mathrm{~g}$ a.i. ha ${ }^{-1}$ fb imazethapyr $(10 \%$ SL) @ $75 \mathrm{~g}$ a.i. $\mathrm{ha}^{-1}$ at $30 \mathrm{DAS}+\mathrm{IC}$ at 60 DAS (8.40 per plate) recorded significantly higher fungal population and were at par with each other. Significantly lower fungal population was noticed quizalofop-ethyl (5\% EC) @ $50 \mathrm{~g}$ a.i. ha ${ }^{-1}$ at 30 DAS + IC at 60 DAS (5.78 per plate) and chlorimuron ethyl (25\% WP) @ 15 g a.i. ha ${ }^{-1}$ at 15-20 DAS + IC at $60 \mathrm{DAS}$ (5.37 per plate.

\section{Soil enzymatic activity}

In the modern day agriculture, a large number of agricultural chemicals are being used to control a wide variety of weeds, pests, and diseases. A judicious and cautious use of these chemicals helps in sustaining the productivity at higher level while their indiscriminate use leads to serious ecological imbalances. The inhibition of soil enzymes by agrochemicals can be direct or indirect. Any action of the chemical altering the life functions of soil organisms could directly affect soil enzyme activity. An agrochemical may also modify the inter relationship between the particular group of organisms thereby influences the amount and type of enzyme produced (Cerevelli et al., 1978). Intensive use of herbicides without adequate knowledge on its effects on soil enzymes may have adverse impact on soil biochemical processes and recycling of nutrients. So, it is important to have knowledge of behavior of herbicides in the soil to avoid soil pollution and their side effects on soil micro-organisms and succeeding crops.

The dehydrogenase enzyme (DHA) is commonly used as an indicator of biological 
activity in soil as well as a direct measure of soil microbial activity. In the present study there has been increased activity of DHA, at lower levels of herbicides. While, there was decreased activity at higher levels. Similarly, in present study there was significant variation from initial and at 60 DAS. Dehydrogenase enzyme activity increased in the plots treated with pendimethalin $(38.7 \%$ CS)@1000 g a.i. ha ${ }^{-1}$ fb imazethapyr (10\% SL) @ $75 \mathrm{~g} \mathrm{a.i.} \mathrm{ha}{ }^{-1}$ at $30 \mathrm{DAS}+\mathrm{IC}$ at 60 DAS (19.42, 23.37 and 17.55 $\mu \mathrm{g}$ TPF formed $\mathrm{g}^{-1}$ soil $\mathrm{hr}^{-1}$ ) at initial, 60 DAS and at harvest (Table 3) and was followed by pendimethalin $38.7 \%$ CS @ $1000 \mathrm{~g}$ a.i. ha ${ }^{-1}$ (PRE) + IC at 30 and 60 DAS (19.38, 23.21 and 17.51 $\mu \mathrm{g}$ TPF formed $\mathrm{g}^{-1}$ soil $\mathrm{hr}^{-1}$ ). It might be due to use of residues of weeds by microbes as a source of carbon for their enzymatic activity. This dehydrogenase activity gradually decreased towards maturity due to soil loss of organic matter due to crop uptake and no more addition of organic matter to soil in the form of dead weeds. Results were in accordance with Younesabadi et al., (2014) who reported that tank-mix application pendimethalin 0.5 $\mathrm{kg} \mathrm{ha}^{-1}+$ imazethapyr $0.075 \mathrm{~kg} \mathrm{ha}^{-1}$ recorded higher bacteria, fungi and actinomycetes population and also increased dehydrogenase activity.

\section{References}

Adhikari, P. and Ghosh, R. K., 2014, Integrated weed management strategies in blackgram-brinjal-mustard cropping sequence. Env. Ecol., 32(2): 725-727.

Casida, L., Klein, D. and Santoro, T., 1964,
Soil dehydrogenase activity. Soil Sci., 98: 371-376.

Cerevelli, S., Nanniperi, P. and Sequi, P., 1978, Interaction between agrochemicals and soil enzymes. In: Soil Enzymes (Ed. Bums, R.G.), Academic Press Inc, New York, pp. 3748.

Devasenapathy, P., Ramesh, R. T. and Gangwar, B., 2008, Efficiency indices for agriculture research. New India Publishing Agency, Pitampera, New Delhi, pp: 65-113.

Dubey, R. C. and Maheshwari, D. K., 2008, Practical Microbiology. S. Chand publications, New Delhi, pp. 86-87.

Gill, B.S., Randhava, G. S. and Saini, S. S., 2002, Integrated weed management in fenugreek (Trigonella foenumgraecum). Indian J. Agron., 47(2): 284288.

Jackson, M. L, 1973, Soil Chemical Analysis, Prentice Hall of India Private Limited, New Delhi, pp. 38-82.

Malik, R. S., Yadav, A. and Malik, R. K., 2008, Evaluation of different herbicides against broadleaf weeds in wheat and their residual effects on sorghum. Indian J. Weed Sci., 40(1\&2): 37-40.

Subbaiah, B. V. and Asija, G. L., 1956, A rapid procedure for the estimation of available nitrogen in soils. Curr. Sci., 25: 259-260.

Younesabadi, M., Das, T. K. and Pual, S., 2014, Tillage and weed management effect on weeds, non-target toxicity in soil and yield of soybean. Int. J. Farm Alied Sci., 3(9): 962-969.

\section{How to cite this article:}

Tasmiya Kowser, A.S. Halepyati, B.M. Chittapur, A.S. Channabasavanna, I. Shanker Goud and Basave Gowda. 2017. Phyllosphere Microflora and Soil Dehydrogenase Activity as Influenced by Weed Management Practices. Int.J.Curr.Microbiol.App.Sci. 6(12): 2473-2479. doi: https://doi.org/10.20546/ijcmas.2017.612.287 